\title{
Two-Dimensional Silicon Vidicon Astronomical Photometer
}

\author{
Thomas B. McCord and James A. Westphal
}

\begin{abstract}
We have developed and successfully used an integrating two-dimensional silicon diode array vidicon photometer which is exceptionally well suited for use with telescopes. The video signal is read out from the $1-\mathrm{cm}^{2}$ silicon target of the vidicon through a current mode preamplifier and then converted to digital form and stored on magnetic tape. The $256 \times 256$ element frames are recorded at 20,000 eight-bit words/sec. The vidicon tube has a published quantum efficiency ranging from $85 \%$ at $0.5 \mu$ to $6 \%$ at $1.1 \mu$ and must be cooled to about $-65^{\circ} \mathrm{C}$ to eliminate thermal dark current. The minimum detectable signal in the present system is about 1000 carriers per resolution element, limited by preamplifier and other system noise. The system is used as a single-frame camera. The large dynamic range $\left(>10^{3}\right)$, linear response, high quantum efficiency over a large spectral region, and low cost of the system make it well suited for digital direct image and spectroscopy as well as for a laboratory digitizer of two-dimensional material.
\end{abstract}

\section{Introduction}

The photographic plate has been the most commonly used two-dimensional detector of telescope images for almost 100 years. During the past five to ten years a variety of electrooptical devices have been discussed as supplements or replacements of photography in astronomy. ${ }^{1}$ Recently, we have developed a twodimensional integrating vidicon photometer which is especially well suited for many types of astronomical observations and which has been mentioned only briefly in the literature. ${ }^{2}$ This article describes the experimental system in its present form.

\section{System}

The photometer consists of four subsystems (see Fig. 1). The heart of the device is a silicon diode array vidicon tube. The video signal is amplified, digitized, and stored on digital magnetic tape by the data system. A shutter mechanism in front of a filter wheel provides the vidicon with an image from the telescope which can also be viewed by the observer through an eyepiece. The vidicon tube is mounted in a dry ice coldbox which is attached to the telescope behind the camera optical system with the remainder of the system mounted in a $53-\mathrm{cm}$ rack attached to the camera head by a cable. The camera head weighs $10 \mathrm{~kg}$ and has approximate dimensions of $20 \mathrm{~cm} \times 20 \mathrm{~cm} \times 40 \mathrm{~cm}$.

\footnotetext{
Thomas B. McCord is with the Planetary Astronomy Laboratory, Department of Earth \& Planetary Sciences, Massachusetts Institute of Technology, Cambridge, Massachusetts 02139; James A. Westphal is with the Division of Geological and Planetary Sciences, California Institute of Technology, Pasadena, California 91103 .

Received 12 October 1971.
}

\section{Vidicon Tube}

The detector is a silicon diode array vidicon tube with conventional direct electron beam readout. ${ }^{3}$ In this tube the vidicon target consists of an array of back-biased silicon diodes which have electrical and optical properties that make this tube especially well suited for telescope photometry. In the target, photons are converted to charge carriers that discharge the backbiased diodes. In the readout mode, the electron beam recharges the discharged diodes creating a current through the target biasing circuit. This current constitutes the video signal.

The target (see Fig. 2) is composed of a wafer of $n$-type silicon with one surface oxidized into which an array of pits have been etched. A $p$-type dopant is diffused through the pits to produce the $p-n$ junctions. Each diode is then covered with an epitaxilly grown silicon top hat, which is physically and electrically isolated from all the others.

In operation, the target is biased to about $10 \mathrm{~V}$, and then the $p$-side of the diode is charged to ground. potential by the electron beam. The diodes are thus backbiased to the target potential. An incoming photon entering the $n$-type silicon target produces a hole-electron charge pair. The hole diffuses across the nearest diode junction and decreases the charge on that diode capacitance by $1.6 \times 10^{-19} \mathrm{C}$. After the exposure is completed the stored image is read out by scanning with the electron beam. The beam recharges the diodes as it sweeps across the target. As a diode is recharged a current flows through the target and creates the video signal in the current mode preamplifier.

Several advantages of the silicon diode tube for photometric observations are evident. First, the conversion of photons to a stored image and the subsequent 


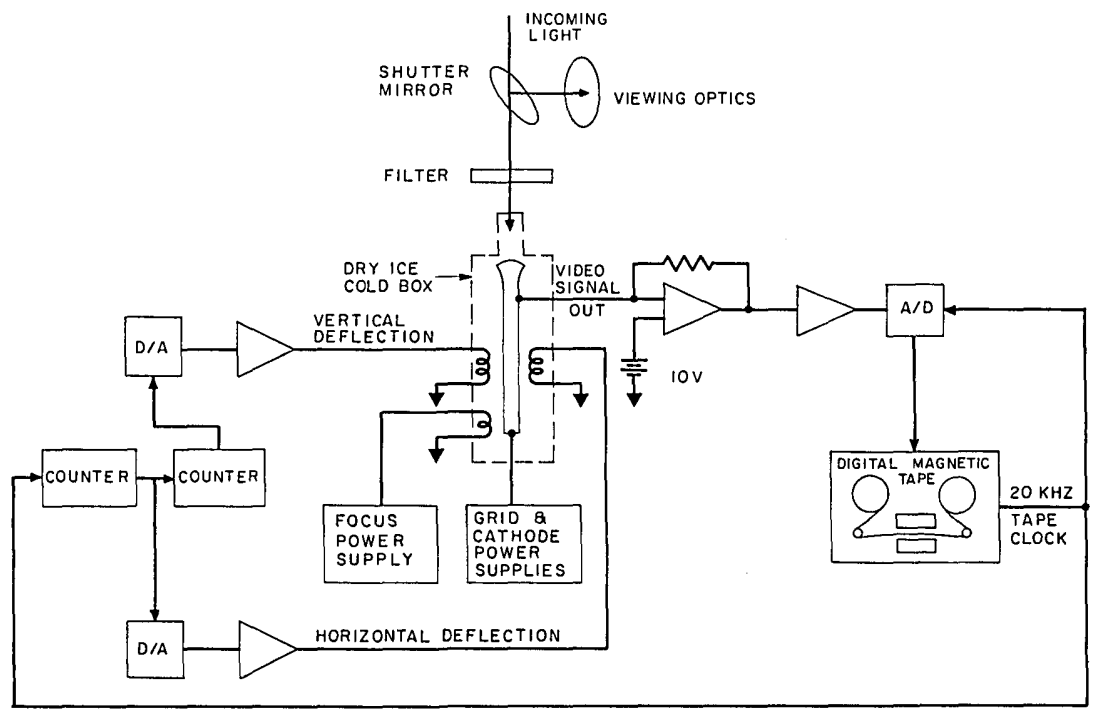

Fig. 1. The entire system is shown schematically.

image readout are linear processes. One photon produces one charge carrier which reaches a diode and neutralizes one charge on the diode capacitance. This linearity results in a $\gamma$ of unity with no reciprocity failures, unlike standard vidicons and photographic film.

Second, sensitivity over a wide spectral range is possible. The red spectral response terminates at about $1.2 \mu \mathrm{m}$ due to the transparency of silicon. Sensitivity at shorter wavelengths appears to be basically limited by the tube envelope material. The published quantum efficiency of the tube we are using (an experimental RCA model C23136) reaches a high of $85 \%$ near $0.50 \mu \mathrm{m}$ and is $6 \%$ at $1.1 \mu \mathrm{m}$ (see Fig. 3) including the effect of an antireflection coating tuned for peak transmission at $5500 \AA$. Thus, the silicon tube is the most sensitive near-ir image detector available at present by several orders of magnitude. The quantum efficiency in this particular tube is limited in the blue by the reflections that take place at the surface of the target and tube envelope and by absorption in the tube envelope as well as by a special surface coating on the target to control carrier recombination. Thicker targets would improve ir sensitivity since practically every photon that is absorbed in the target creates a charge carrier. However, the spatial resolution would be decreased because of the increased sideways charge carrier diffusion in the thicker target. Infrared peaked antireflection coatings would also increase the effective quantum efficiency.

The ultimate spatial resolution of the silicon tube is limited by the discrete nature of the target. Our target has $15-\mu \mathrm{m}$ diode spacings, and $10-20-\mu \mathrm{m}$ spacing is normal for most tubes available. One could achieve maximum resolution by focusing the electron read beam to a spot smaller than one diode. However, a complicated MTF results at high spatial frequency from the regularity of the discrete diode array and from any lack of registration of the read beam raster with the diode array resulting in a moiré pattern.
We have chosen to operate with a defocused read beam that gives us about 256 nonoverlapping samples across a 1-cm target. We do this because we do not require higher spatial resolution for current projects and because it simplifies data handling. The tube manufacturer quotes the basic spatial square wave response to be that given in Fig. 4 . In the future, we intend to investigate the problems of achieving the highest spatial resolution.

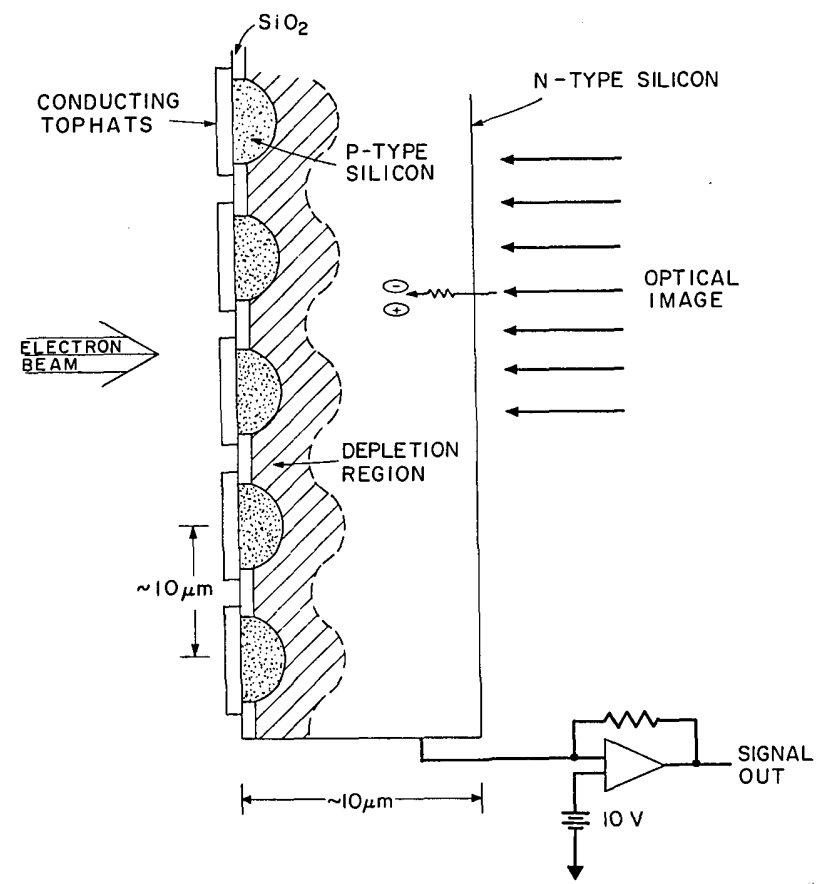

Fig. 2. The target of the vidicon is shown idealized. Photons incident produce charge pairs. The hole diffuses across the nearest back-biased diode and discharges the diode capacitance. The electron read beam replaces the charge on the diode, creating a current flow in the video circuit. The target thickness can be increased for greater ir sensitivity at the expense of lower spatial resolution due to lateral diffusion of charges. 


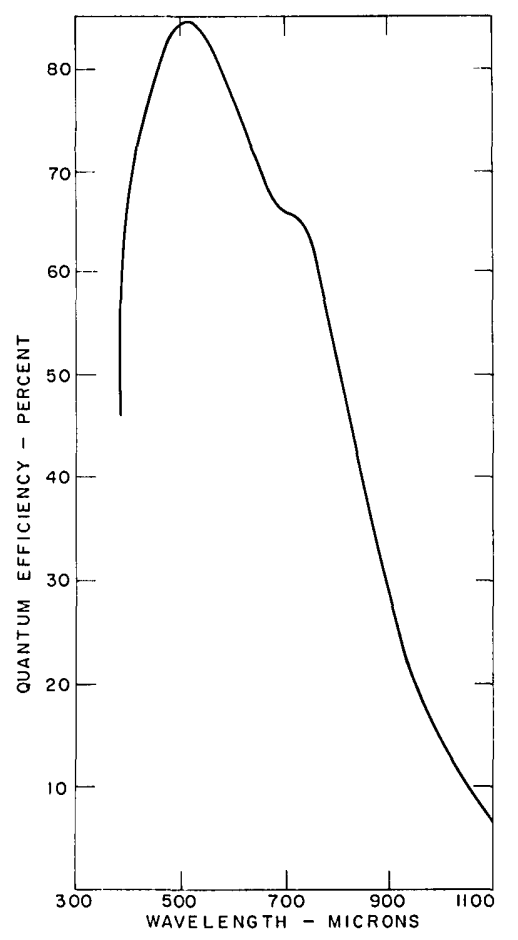

Fig. 3. The published spectral response of the commercial grade RCA silicon vidicon including the effects of an antireflection coating peaked for maximum transmission at about $0.5 \mu \mathrm{m}$.

\section{Camera}

The silicon diode-array tube is operated like a standard vidicon, using magnetic focus and deflection. The tube and the precision slow-scan deflection yokes are mounted in an insulated box which is cooled with dry ice. The power supplies and electronics are located in a rack and are attached to the camera head by one master cable.

The horizontal and vertical deflection is digitally controlled using either an external clock or the internal clock controlling the magnetic tape recorder (see Fig. 1). In normal read mode the $20-\mathrm{kHz}$ tape recorder clock is fed to an eight-bit binary counter driving a D/A converter. The $\mathrm{D} / \mathrm{A}$ converter produces a stepped ramp function which is amplified to drive the horizontal deflecting yoke. Before driving the yoke the stepped ramp is smoothed to produce a continuous scan pattern. The length of the horizontal scan is 256 steps. The counter overflow at 256 is used to drive a second eight-bit binary counter which feeds another D/A converter driving the vertical deflection yoke through another amplifier. With the $20-\mathrm{kHz}$ basic clock the entire $256 \times 256$ frame is scanned in approximately 3.3 sec. The camera is usually read out in the single frame mode, but a continuous mode is available for erase and setup monitoring.

A shutter mechanism and field viewing optics are built into the camera head. Interference filters, 2.5 $\mathrm{cm}$ in diameter, are mounted in a filter wheel directly in front of the cold-box window.

\section{Data System}

The video signal is created by the electron beam recharging the silicon diodes on the tube target. As charge is replaced on the diode capacitance a current

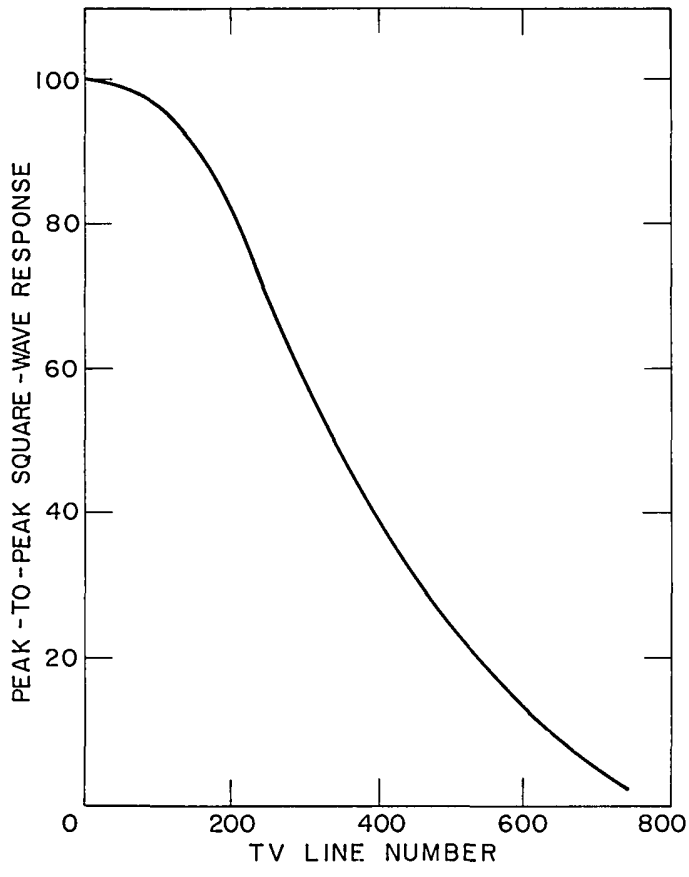

Fig. 4. The smoothed square wave response function for the silicon vidicon as given by RCA.

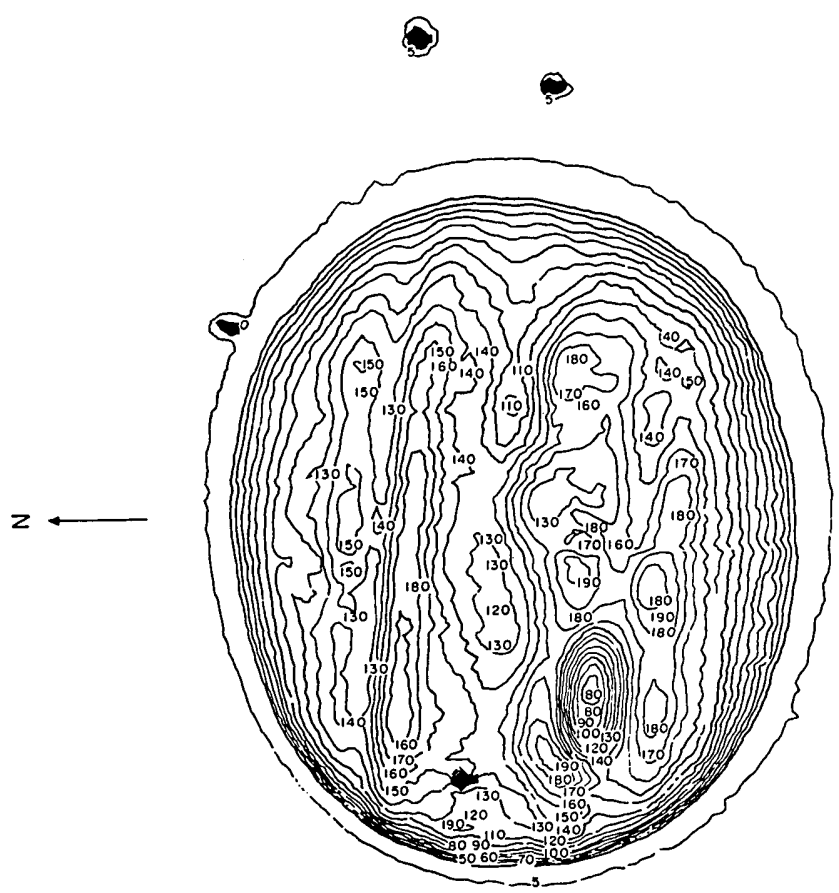

Fig. 5. Intensity contours of an image of Jupiter made through a $300-\AA$ wide interference filter centered at $0.40 \mu \mathrm{m}$. These are raw data unprocessed for noise or target sensitivity. The small intense areas near Jupiter's west limb are due to defective diodes on the target. Larger numbers $=$ lighter areas. The solar phase angle is about $12^{\circ}$ so the eastern terminator is not visible. The Great Red Spot appears as a very dark area in the southeast quadrant. Note the intense uv bright spots near the GRS. The contours near the limb have been omitted except for the last one. This image and that in Fig. 6 were made using the $152-\mathrm{cm}$ telescope of the Cerro Tololo Interamerican Observatory, Chile. 
flows in the target bias circuit (see Fig. 1). The video preamplifier, operating in a current mode, produces an analog voltage that is proportional to the amount of charge required to recharge the diode on the particular part of the target accessed by the electron beam. The video voltage signal is further amplified and is then filtered by a low pass filter at the input of an A/D converter. The $A / D$ converter is controlled by the magnetic tape recorder clock to sample at every clock pulse $(20 \mathrm{kHz})$ while the electron beam is scanning the target.

Notice that both the scan electronics and the data system are synchronized to the tape recorder. The recorder runs continuously at $20 \mathrm{kHz}$ for rapid readout with one clock pulse resulting in one horizontal step of the video scan and in one sample of the video signal.

The tape recorder is a Cipher Data Products ninetrack machine on which we write one eight-bit data word per sample.

\section{Operation}

The system is operated as a single frame, slow scan digital camera. To begin operation the camera head is mounted on the telescope and cooled to thermal equilibrium with dry ice. After initial checkout, an image to be recorded is placed on the reticle of the viewing optics. A filter of the desired wavelength and passband is selected, and the exposure time is chosen and set. The vidicon tube is then erased by multiple scans of the electron beam. At the low beam current used for the slow scan readout mode about four to six erasure scans are needed to erase completely the target after a saturated exposure, since after the first recording of the image about $10 \%$ of the image is retained by the target. This is probably due to a poorly understood form of charge storage occurring on the back of the target and not to any photoconduction lag in the target.

With the target erased, scanning is stopped, and the exposure is begun by opening the shutter. If the exposure is to be longer than a few minutes, the filament in the tube is turned off to prevent backlighting of the target. For exposure of $\frac{1}{2} \mathrm{~h}$ or longer all tube power, except the target voltage, is turned off to reduce the heat load in the cold box.

At the end of the exposure the shutter is closed, and the tube power and the filament are turned on to prepare for readout. The image is read off the target by a single raster scan of the electron beam. The image is recorded on a magnetic tape as 65,536 eight-bit words and, in parallel, on Polaroid film using an oscilloscope.

The magnetic tapes are computer processed to produce the images in various forms for analysis. At the present time routine digital image processing is performed on our in-house computing facilities. The linearity and stability of the vidicon tube make such necessary processes as photometric calibration, contrast enhancement, and frame comparison straightforward.

\section{Performance}

We have taken over 700 frames at the telescope and additional frames in the laboratory using the vidicon photometer as described above. The performance of the system has been just what one would expect from the vidicon tube characteristics and the system parameters we have chosen. Of particular interest to astronomers are the sensitivity of the tube in actual operation and the performance of the tube during long integrations.

The over-all sensitivity of the system is a function of both the tube quantum efficiency (see Fig. 2) and the system noise. At present we are limited to detecting about 1000 carriers per resolution element because of amplifier and system noise. We feel that as much as an order of magnitude improvement in SNR is possible. From our measurements of 47 Tucanae one should detect stars of eighteenth magnitude with a SNR of $5: 1$ in an integration period of 100 sec using a 152-cm telescope, no filter (other than the glass tube envelope), and our beam size and scan speed.

We have used the present system to integrate on a scene at the telescope for as long as $1 \mathrm{hr}$. It is necessary to cool the tube to reduce thermally induced charge carriers in the target which can discharge the diodes (dark current). We find a 5\% loss in our present dynamic range of $10^{3}$ in $1 \mathrm{~h}$ of integration with a target temperature of $-67^{\circ} \mathrm{C}$ because of dark current. This agrees with theoretical calculations. Cooling to a lower temperature should improve this figure even more. There appear to be no adverse effects on the tube performance due to cooling.

An isophoto contour map of one frame made using the photometer is shown in Fig. 5. The image is of Jupiter taken through a $300-\AA$ wide interference filter centered at $0.4 \mu$. The 152-cm telescope at the Cerro Tololo Interamerican Observatory in Chile was used. These data are from unprocessed numbers taken directly from the magnetic tape recorded at the telescope and have not been corrected for flat field response. A 1min exposure of the central 100 sec of arc region of 47 Tuc is shown in Fig. 6. The faintest stars contoured are around eighteenth magnitude.

\section{Applications}

The device described here has been used for photometry at the telescope. It is obvious that equally important results can be achieved by using it to record spectra at the telescope. In addition, there are many laboratory applications where two-dimensional analog data (such as old photographic plates) might be digitized. This system would accomplish the conversion very simply.

We are grateful for the considerable aid received from Eugene Gordon of Bell Telephone Laboratories, Murray Hill and Kenneth Ando of Jet Propulsion Laboratory, Pasadena, and their co-workers. Their discussions were invaluable to the success of this project. Jay Kunin of MIT was a dedicated participant during the 


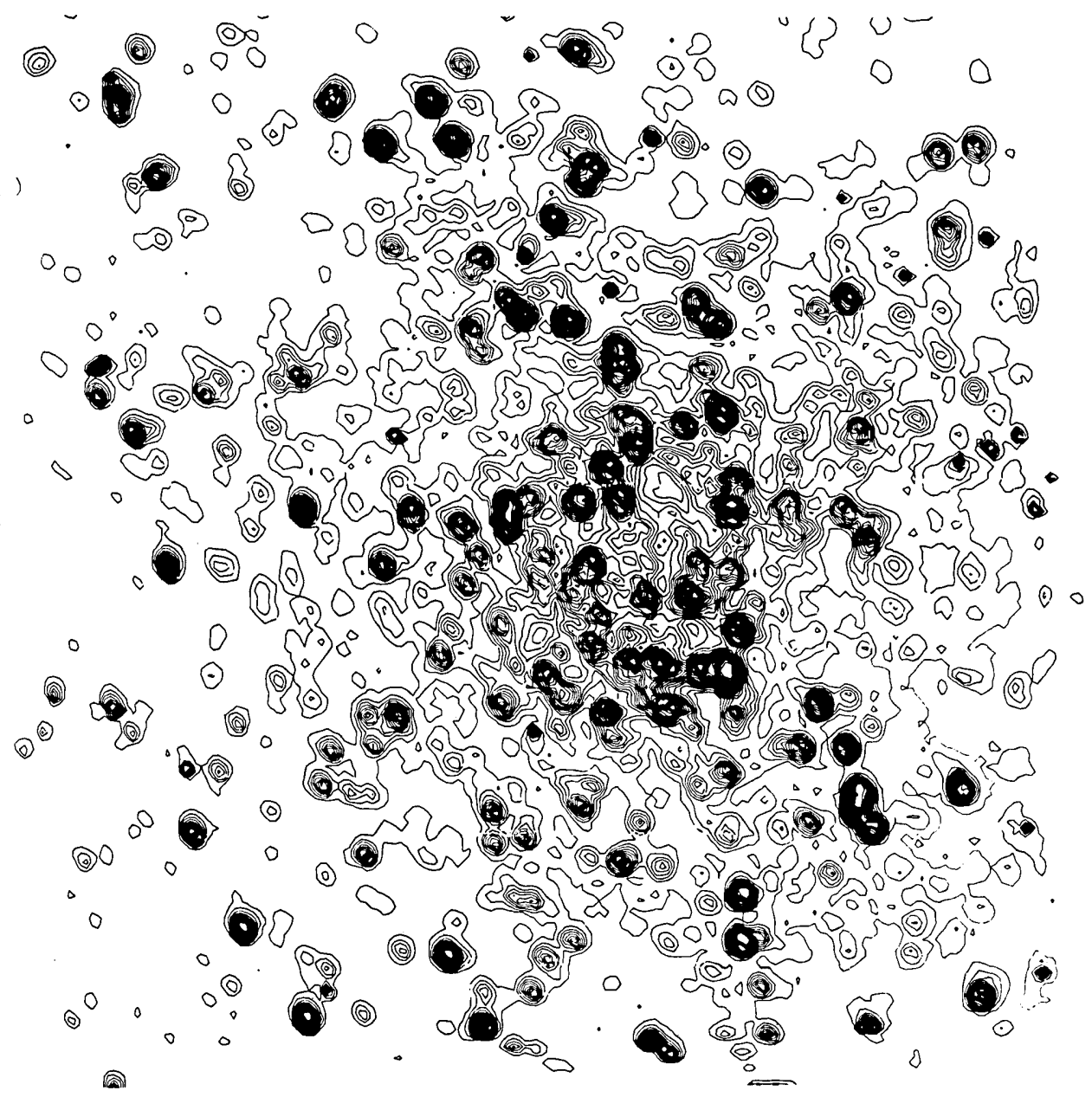

Fig. 6. Intensity contours of a 1-min unfiltered exposure of the central $100 \mathrm{sec}$ of arc region of the globular star cluster 47 Tucanae. The faintest stars contoured are about eighteenth magnitude.

final stages of development. Special thanks go to William Thompson of Bellcomm, Washington, D.C., who first called our attention to the silicon target vidicon and to Frank Press of MIT who enabled us to act on the idea early.

Telescope time for the first use of the system was made available by the Cerro Tololo Interamerican Observatory, Chile, an NSF sponsored facility.

This paper is Contribution 44 of the Planetary Astronomy Laboratory and contribution 2074 of the Division of Geological and Planetary Sciences.

\section{References}

1. L. M. Biberman and S. Nudelman, Eds., Photoelectronic Imaging Devices (Plenum, New York, 1971).

2. J. A. Westphal and T. B. McCord, "A Silicon Vidicon Photometer," presented at the Symposium, Advanced Electronic Systems for Astronomy, Santa Cruz (31 August-2 September 1971). Proceedings to appear in Publ. Astron. Soc. Pacific.

3. M. H. Crowell and E. F. Labuda, in Photoelectronic Imaging Devices, L. M. Biberman and S. Nudelman, Eds. (Plenum New York, 1971), Vol. 2.

\title{
CHANGES OF ADDRESS
}

\section{ZIP CODES}

\begin{abstract}
Changes of address should be sent to Appuied Optics SUBSCRIPTION HANDLING, American Institute of Physics, 335 East 45th Street, New York, New York 10017, as far in advance as possible. Eight weeks should be allowed to effect such change.

Please confirm your zip code next time you change your address or you renew your subscription.
\end{abstract}

